\section{Recommendations for the analysis of hemocyte-related immunocompetent oxidative activity in the freshwater snail Lymnaea stagnalis}

\author{
P. Boisseaux, ${ }^{1}$ P. Noury, ${ }^{1}$ \\ M.-L. Delignette-Muller, 2,3 \\ H. Thomas, ${ }^{4}$ J. Garric ${ }^{1}$
}

'Irstea, UR MALY, Centre de LyonVilleurbanne, Villeurbanne; 'Université de Lyon, Université Lyon 1, CNRS, UMR

5558, Laboratoire de Biométrie et Biologie Evolutive, Villeurbanne; 3Université de Lyon, VetAgro Sup Campus Vétérinaire de Lyon, Marcy l'Etoile; ${ }^{4}$ LIttoral ENvironnement et Sociétés (LIENSs) - UMR 7266, La Rochelle, France

\section{Introduction}

One of the most remarkable features of immune systems through many species is the capacity of phagocytes to produce diverse form of oxidizing agents in order to destruct invaders. Reactive oxygen species (ROS) represent a family of various reactive molecules that play pivotal roles in a myriad of physiological and pathophysiological events. The NADPH oxidase catalytic complexes (also called respiratory burst oxidases) are most often admitted to be responsible for the hemocyte immunocompetent oxidative activity. ${ }^{1-3}$ These enzymes are activated when the cell is exposed to appropriate stimuli such as pathogen-associated-molecular patterns (PAMPs): the phenomenon is inducible. ${ }^{4,5}$ In several mollusks, the phagocytosis of microorganisms promotes the activation of the NADPH oxidases from hemocytes, ${ }^{6-9}$ in particular in Lymnaea stagnalis. ${ }^{10}$ In L. stagnalis, it has been observed that repeated hemolymph samplings at a $48 \mathrm{~h}$ frequency did not affect the snails immunocapacity defined as the combination of hemocyte density and hemocyte viability. ${ }^{11}$ However, other endpoints may still be affected such as functional markers for example, as it was reported for lysosomal enzymatic activity in L. stagnalis. ${ }^{12}$ The aims of this study was: i) to develop a method in order to follow kinetics of hemocyte-related oxidative activities under basal and immunostimulated $e x$ vivo conditions; and ii) to assess possible effects of repeated hemolymph collections on hemocyte oxidative activities.

\section{Materials and Methods}

A total of 24 calibrated snails $(32 \pm 2 \mathrm{~mm}$ shell-length), free of parasites, were taken from the breeding stock of our laboratory and randomly assigned into three aquaria. Snails were maintained in a flow-through system of clean groundwater as for breeding stocks $\left(20 \pm 1^{\circ} \mathrm{C}, 16 \mathrm{~h} / 8 \mathrm{~h}\right.$ of light/dark cycles, ad libitum feeding with Tetraphyll(C). The experiment duration lasted for four days and the hemolymph of individual snails was collected mechanically as described by Sminia ${ }^{13}$ and at three times: at $\mathrm{t}=0 \mathrm{~h}, \mathrm{t}=48 \mathrm{~h}$ and $\mathrm{t}=96 \mathrm{~h}$. When tickling the foot sole of the snail with the tip of a micropipette, the snail retracts into its shell and extrudes hemolymph through the hemal pore. The methodology of ROS measurement was adapted from Adema, van DeutekomMulder, ${ }^{10}$ Moss and Allam ${ }^{14}$ and Hahn, Bender. ${ }^{9}$ For each snail, $25 \mu \mathrm{L}$ of hemolymph were gently deposited into 12 wells of a 96-well plate (treated for cell culture, Greiner, Cat. No.

655180). The plates were centrifuged (100 g, $20 \mathrm{~min}$ ), the lymph was removed and then replaced by snail saline buffer solution (SSB), ${ }^{15}$ pre-warmed at room temperature, to

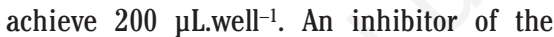
NADPH-oxidase, namely the protocatechuic acid (PCA), ${ }^{9,10,16}$ was used for the half of the wells $(160 \mu \mathrm{M})$. After $30 \mathrm{~min}$ of slow agitation on a shaker, the half of these two groups of wells $(\mathrm{N}=24$ triplicates per organism per $e x$ vivo condition) was supplemented with zymosan particles $\left(50 \mu \mathrm{L}\right.$ of a $2 \mathrm{mg} \cdot \mathrm{mL}^{-1}$ of SSB working solution) (Sigma Z4250), which are PAMPs from yeasts (Saccharomyces cerevisi$a e$ ). The non-stimulated cells received $50 \mu \mathrm{L}$ of SSB. A stock solution of the cell-permeant probe 2',7'-dichlorodihydrofluorescein diacetate ( $\left.\mathrm{H}_{2} \mathrm{DCFDA}\right)$ (D6883 Sigma) was previously prepared in ethanol $(20 \mathrm{mM})$ and conserved at $-20^{\circ} \mathrm{C}$. Straightforwardly after zymosan and SSB additions, the $\mathrm{H}_{2}$ DCFDA stock solution was diluted at $2 \mathrm{mM}$ using SSB and then $10 \mu \mathrm{L}$ were added into each well. Samples were homogenized and then the plate was read using a spectrofluorimeter (TECAN, Safire). The fluorescence at $485 / 530 \mathrm{~nm}$ (EX/EM) wavelengths was measured for $1 \mathrm{~h}$ every $2 \mathrm{~min}$. First, the raw data of relative fluorescence obtained from kinetics were converted into amount of converted product DCF (Dichlorofluorescein) by using a standard curve. Second, the activity was calculated as the mean of triplicates for each ex vivo condition from each snail, according to the formula:

Oxidative activity (fmol of $D C F / \min / \mu L$ )

$=\frac{\text { slope } X i j(20-40 \mathrm{~min})}{25 \mu \mathrm{L}}$
Correspondence: Jeanne Garric, Irstea, UR MALY, centre de Lyon-Villeurbanne, 5 rue de la Doua, BP 32108, 69616 Villeurbanne Cedex, France.

E-mail: jeanne.garric@irstea.fr

Key words: Lymnaea stagnalis; hemocyte-related oxidative activity; immunocompetence.

Funding: the Rhône-Alpes region provided financial support to Paul Boisseaux (IRC3 Environnement).

Conference presentation: part of this paper was presented at ECOBIM meeting, 2016 May-June, Le Havre, France.

This work is licensed under a Creative Commons Attribution NonCommercial 4.0 License (CC BYNC 4.0).

(C) Copyright P. Boisseaux et al., 2016

Licensee PAGEPress, Italy

Journal of Xenobiotics 2016; 6:6585

doi:10.4081/xeno.2016.6585

where $X$ is the amount of DCF (fmol) at the minute $i$ for the snail $j$.

The datasets were analyzed by fitting linear mixed-effects models (LMEM) using the lmer function from the lme 4 package ${ }^{17}$ in the R software. Beforehand, we applied a $\log _{10}$ transformation to the data to normalize the distributions. LMEM were first built with three fixed factors, the zymosan (i.e., presence/absence), the PCA (i.e., presence/absence), and the day, and two nested random factors, the snail and the replicate (aquarium). The initial model also included interaction terms between zymosan and PCA, zymosan and day, PCA and day. The model was simplified by removing the effects that did not appear significant. An effect was considered as statistically significant when the $95 \%$ confidence interval of any of its corresponding coefficients did not contain the value 0 .

\section{Results and Discussion}

\section{Baseline activities}

In the present study, the data are expressed in fmol of DCF.min ${ }^{-1} . \mu \mathrm{L}^{-1}$. It corresponds to the oxidative activity from hemocytes (i.e., the lymph was removed) per microliter of hemolymph. The transformation of relative fluorescent units in amount of substance converted has been made in order to facilitate the data comparisons between independent experiments and the use of different spectrofluorimeter settings (e.g., gains). By using the corresponding standard curve, the same data calculation is possible. The underlying question that justified such a methodology was: what is 
the hemocyte-related immunocompetent oxidizing ability in the circulating system of the snail? Indeed, interpretation of ROS productions are tricky in immunophysiopathology because they encompass many origins (e.g., mitochondrial, endoplasmic reticulums, peroxisome activities, etc.), ${ }^{3}$ NADPH-activity, ${ }^{10}$ mechanical-stress ${ }^{18}$ and xenobiotic-oxidative stress $^{15,19,20}$ among others. They may constitute indication of toxicity or of immunocompetence (oxidative stress vs immunocompetent oxidative activity) and so basal together with stimulated activities are both complementary. Last, the lymph was removed in order to reduce ROS background coming from other pathways than hemocytes.

ROS production showed a strong increase when the samples were stimulated with zymosan and a strong decrease when they were incubated with PCA (Figure 1). A transient reduction phase occurred at approximately 25 min (Figure 2). Afterwards, ROS production increased linearly. The mean (in decimal logarithm) at day 0 for samples without zymosan and without PCA was estimated to 2.39 [with $95 \%$ confidence interval (CI) of (2.26-2.51)]. This $\log _{10}$ value corresponds to a hemocyte-related oxidative activity of 245.47 fmol of DCF.min ${ }^{-1} \cdot \mu^{-1}$. In average, the immunostimulation with zymosan increased significantly the $\log _{10}$ value of the basal activity by 0.82 (95\% CI of $0.74-0.90)$, which corresponds to an increase by a factor 6.61 of the activity $\left(10^{0.82}=6.61\right)$. The presence of the inhibitor PCA decreased significantly the $\log _{10}$ value of the basal activity by $0.23(95 \% \mathrm{CI}$ of $0.19--0.25)$. A significant interaction was found between zymosan and PCA. Hence, the presence of PCA diminished by $0.53(-0.53=-$ $0.23-0.30$ ) the $\log _{10}$ values of the zymosanstimulated oxidative activity instead of 0.23 in the absence of zymosan. Addition of PCA did not lead to a proportional inhibitory effect whether the samples were immunostimulated with zymosan or not.

\section{Repeated collections}

Data analysis from the model revealed a significant effect of the day 4 but not the day 2 compared to the day 0 . The oxidative activities at day 4 were estimated to multiply by 0.64 the ones from day 0 . In other words, the third hemolymph collection led to a significant decrease in the oxidative activities (i.e., basal and immunostimulated) of approximately $36 \%$ from the oxidative activities measured at day 0 . No significant interactions were found neither between the day and the zymosan nor between the day and the PCA. The repeated hemolymph collections decreased both basal and zymosan-stimulated oxidative activity from hemocytes. The decreases are particularly important for the third hemolymph collection (i.e., day 4) on Figure 1. Such phenome-

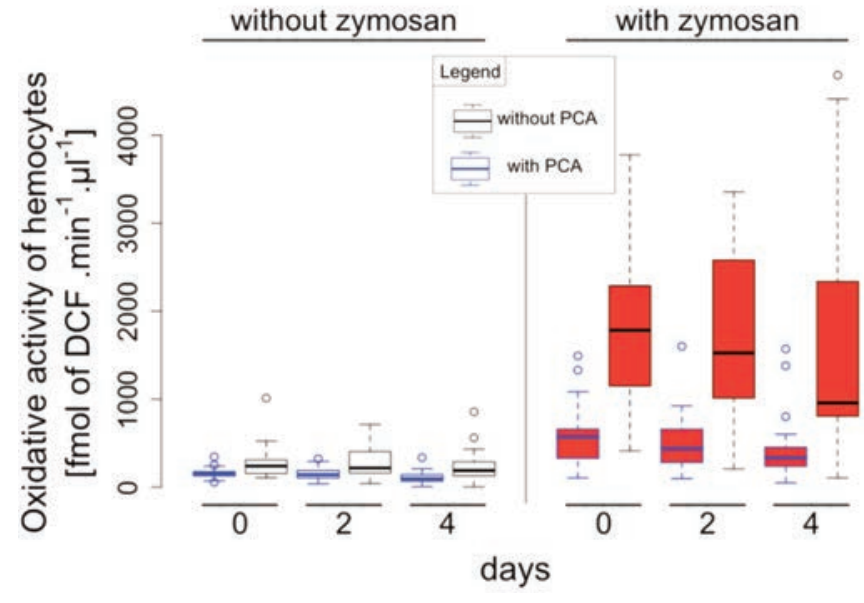

Figure 1. Hemocyte-related oxidative activities under repeated hemolymph collections. The graph represents the oxidative activity from snail's hemocytes - being sampled every $48 \mathrm{~h}$ - under different ex vivo conditions. The ex vivo conditions correspond to samples with zymosan (right) and without zymosan (left); both groups were supplemented with PCA (blue borders) or not (black borders).
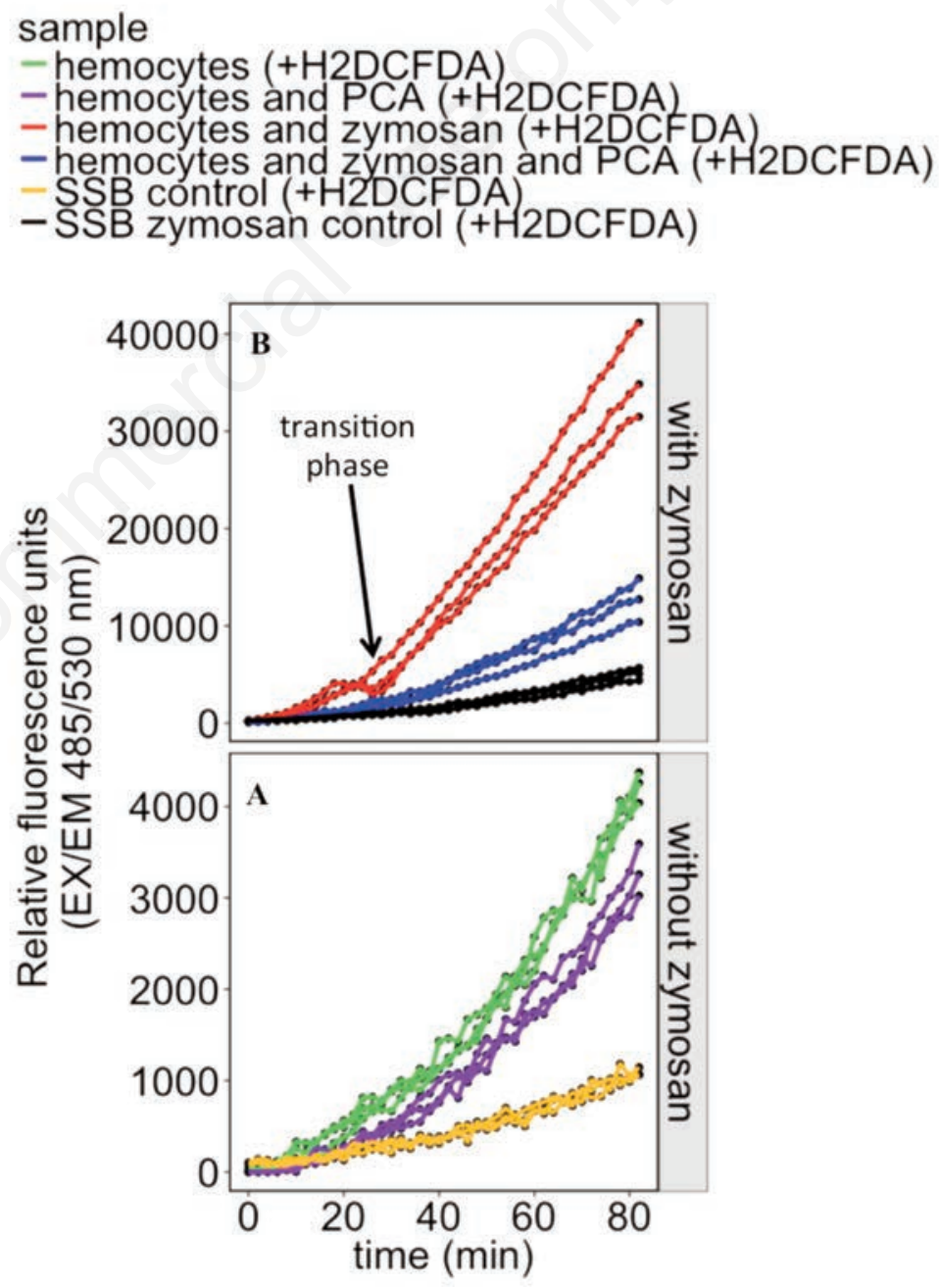

Figure 2. Example of kinetics of hemocyte-related oxidative activities from $25 \mu \mathrm{L}$ of hemolymph [gain $=130$ ]. The graph represents an example of the ROS kinetics obtained from raw data with the spectrofluorimeter into the different ex vivo conditions, for one snail, with corresponding controls. The black arrow indicates a kinetic transition (i.e., unknown phenomenon) being observed in most of the snails. Note that the scale from graph B is ten times bigger than the one from the graph A (values are much higher with zymosan particles and hemolymph). 
non could be the result of a shift in hemocytes maturity (linked to hemocyte renewal turnover) provoked by repeated collections and so young hemocytes would not have the cell machinery - or to a lesser extent - to carry out the immunocompetent oxidative activity. Further studies should be conducted to confirm or infirm this hypothesis. In parallel, at each sampling time the snails' hemocyte density and viability have been quantified by flow cytometry but no sampling effect has been detected on these endpoints.

\section{Conclusions}

The presented method has been optimized because: i) only $25 \mu \mathrm{L}$ of hemolymph per well replicate are necessary and so it is possible to set up several well replicates per individual; ii) spectrofluorimetry allows assessing real kinetics of a broad number of samples, what is tricky in flow cytometry; iii) lymph is removed and so the ROS production originates mainly from hemocytes; iv) zymosan particles are strong inductors of the immunocompetent oxidative activity; v) linear mixed-effect models used allow quantifying the effect of all factors, including time.

We recommend increasing the time interval (i.e., more than $48 \mathrm{~h}$ ) if more than two consecutive hemolymph collections are performed on one snail, otherwise the effect of mechanical hemolymph collection will likely impact the hemocyte oxidative activities and could lead to a bias in results interpretation.

\section{References}

1. Bedard K, Krause K-H. The NOX family of ROS-generating NADPH oxidases: physiology and pathophysiology. Physiol Rev 2007;87:245-313.

2. Buggé DM, Hégaret H, Wikfors GH, Allam B. Oxidative burst in hard clam
(Mercenaria mercenaria) haemocytes. Fish Shellfish Immun 2007;23:188-96.

3. Donaghy L, Hong H-K, Jauzein C, Choi K$\mathrm{S}$. The known and unknown sources of reactive oxygen and nitrogen species in haemocytes of marine bivalve molluscs. Fish Shellfish Immun 2015;42:91-7.

4. Babior BM, Benna JE, Chanock SJ, Smith RM. The NADPH oxidase of leukocytes: the respiratory burst oxidase. Cold Spring Harbor Monogr Archiv 1997;34:737-83.

5. Donaghy L, Kraffe E, Le Goïc N, Lambert C, Volety AK, Soudant P. Reactive oxygen species in unstimulated hemocytes of the Pacific oyster Crassostrea gigas: a mitochondrial involvement. PLoS One 2012;7:e46594.

6. Lambert C, Soudant P, Jegaden M, Delaporte M, Labreuche Y, Moal J, et al. In vitro modulation of reactive oxygen and nitrogen intermediate (ROI/RNI) production in Crassostrea gigas hemocytes. Aquaculture 2007;270:413-21.

7. Manduzio H, Rocher B, Durand F, Galap C, Leboulenger $\mathrm{F}$. The point about oxidative stress in molluscs. ISJ 2005;2:91-104.

8. Bugge DM, Hegaret H, Wikfors GH, Allam B. Oxidative burst in hard clam (Mercenaria mercenaria) haemocytes. Fish Shellfish Immunol 2007;23:188-96.

9. Hahn UK, Bender RC, Bayne CJ. Production of reactive oxygen species by hemocytes of Biomphalaria glabrata: carbohydrate-specific stimulation. Develop Comparat Immunol 2000;24:531-41.

10. Adema C, van Deutekom-Mulder E, van der Knaap W, Sminia T. NADPH-oxidase activity: the probable source of reactive oxygen intermediate generation in hemocytes of the gastropod Lymnaea stagnalis. J Leuk Biol 1993;54:379-83.

11. Boisseaux P, Delignette-Muller M-L, Abbaci K, Thomas H, Garric J. Analysis of hemocytes in Lymnaea stagnalis: characterization and effects of repeated hemolymph collections. Fish Shellfish Immun 2016 [Epub ahead of print].

12. Mohandas A, Adema C, Van der Knaap W,
Sminia T. The effect of haemolymph extraction on distribution of lysosomal enzymes in Lymnaea stagnalis haemocytes: a cytochemical study. Comparat Haematol Int 1992;2:61-7.

13. Sminia T. Structure and function of blood and connective tissue cells of the fresh water pulmonate Lymnaea stagnalis studied by electron microscopy and enzyme histochemistry. Zeitschr Zellforsch mikroskop Anat 1972;130:497-526.

14. Moss B, Allam B. Fluorometric measurement of oxidative burst in lobster hemocytes and inhibiting effect of pathogenic bacteria and hypoxia. J Shellfish Res 2006;25:1051-7.

15. Russo J, Lefeuvre-Orfila L, Lagadic L. Hemocyte-specific responses to the peroxidizing herbicide fomesafen in the pond snail Lymnaea stagnalis (Gastropoda, Pulmonata). Environ Pollut 2007;146:4207.

16. A't Hart B, Simons JM, Rijkers GT, Hoogvliet JC, Van Dijk H, Labadie RP. Reaction products of 1-naphthol with reactive oxygen species prevent NADPH oxidase activation in activated human neutrophils, but leave phagocytosis intact. Free Radical Biol Med 1990;8:241-9.

17. Bates D, Maechler M, Bolker B, Walker S. Fitting linear mixed-effects models using \{lme4\}. J Stat Softw 2015;67:1-48.

18. Lacoste A, Malham SK, Gélébart F, Cueff A, Poulet SA. Stress-induced immune changes in the oyster Crassostrea gigas. Dev Comparat Immunol 2002;26:1-9.

19. Winston GW, Moore MN, Kirchin MA, Soverchia C. Production of reactive oxygen species by hemocytes from the marine mussel, Mytilus edulis: lysosomal localization and effect of xenobiotics. Comparat Biochem Physiol Part C 1996;113:221-9.

20. Koutsogiannaki S, Franzellitti S, Fabbri E, Kaloyianni M. Oxidative stress parameters induced by exposure to either cadmium or 17 -estradiol on Mytilus galloprovincialis hemocytes. The role of signaling molecules. Aquat Toxicol 2014;146:186-95. 